\title{
Aspectos históricos dos estudos caso-controle
}

\author{
Historical issues in case-control studies
}

Marco Antônio Vasconcel os Rêgo 1

\footnotetext{
1 Centro de Estudos da Saúde do Trabal hador, Secretaria da Saúde do Estado da Bahia. Rua Pedro Lessa 123, Salvador, BA 40110-050, Brasil. mrego@lognet.com.br
}

Abstract This paper presents the main issues in the evolution of case control studies (CCS) as of the late 1980s. The comparison of two groups regarding exposure to a risk factor has been known since the 18th century. The latter half of the 19th century witnessed a decline in the "Epidemiology of Populations", and the first CCS were only done in the 1920s. Improvement of the method occurred in the latter half of the 20th century, related to increased mortality from chronic diseases, especially in research on lung cancer and smoking. The main scientific contributions to the method were: use of the odds rati o as an estimate of relative risk; definition of statistical data analysis for retrospective studies; calculation of attributed risk and etiologic fraction; and discussion of the essence of CCS. Critics pointed to the method's possible weakness and susceptibility to bias. Application of CCS increased in the final decades of the 20th century in various areas of Epidemiology and has been considered an important tool to improve Public Health.

Key words Epidemiologic Methods; Case-Control Studies; Public Health Practice

Resumo Este texto apresenta um rel ato da evolução dos estudos de caso-controle (ECC) até o final dos anos 80. A comparação de dois grupos quanto à exposi ção a um fator de risco é verificada desde o século XVII. A segunda metade do século XIX significou o declínio da Epidemiologia das "populações", e os primeiros ECC só foram realizados na década de 20. O avanço do método ocorreu na segunda metade do século, com destaque para as investi gações sobre câncer de pulmão e hábito de fumar. As prin cipais contribuições dos estudiosos do método foram o uso da odds ratio como estimativa do risco rel ativo; a defi ni ção dos aspectos estatísticos da análise de dados de estudos retrospectivos; o cál culo do risco atri buí vel e da fração etiológi ca para ECC; ea discussão da essência dos ECC. Os críti cos referiam as fragi lidades do método e a susceptibi lidade aos bias. Conclui-se que osECC tiveram aplicação crescente nas últimas décadas, sendo utilizados em diversas áreas da epi demiologia, constituindo-se em um importante instrumento para as ações de Saú de Pública.

Palavras-chave Métodos Epidemi ológicos; Estudo de Caso e Controles; Práticas de Saú dePública 
Introdução

Os estudos de caso-controle (ECC) são de desenvolvimento relativamente recente dentro do processo de evolução da epidemiologia (Wünsch Filho, 1992). Inicialmente, os resultados gerados por ECC eram apenas uma indicação, vistos como preliminares, e a prova da associação dependia da realização de um posterior estudo de coorte (Acheson, 1979). Cole (1979) aval iou as publicações de duas revistas médicas e de duas da área de epidemiologia, entre meados de 1950 e meados de 1970, e constatou que o número de ECC publicados cresceu de quatro a sete vezes, o que equivale a dizer que neste segundo momento, os ECC já se constituíam no principal método utilizado nas pesquisas médicas e epidemiológicas.

Mais utilizados em investigações que envolvem as doenças crônicas, os ECC têm tido aplicação crescente nas últimas décadas. As variadas formas de aplicação no campo da epidemiologia e da saúde pública vão além da pesquisa etiológica, e seguem na busca de solução para problemas específicos na prática cotidiana da saúde pública e da medicina (Armenian \& Lilienfeld, 1994). Bons exemplos são a sua utilização na avaliação de medidas preventivas em geral ou utilização de screening (Clarke \& Anderson, 1979; Tsai et al., 1995; Weiss, 1994), na eficácia de vacinas (Comstock, 1994; Noronha et al., 1995; Rodrigues, 1986, apud Wünsch Filho, 1992) ou para avaliação de serviços de saú de. Nestes últimos são definidos como grupo-caso indivíduos com pouco sucesso no tratamento de uma dada doença para a qual já existem alternativas terapêuticas eficazes, e como grupo-controle, indivíduos com resultado satisfatório do tratamento (Buck, 1979; Cole, 1979; Selby, 1994).

O termo caso-controle foi inicial mente proposto por Sartwell em 1960 (Cole, 1979). Este autor refere o problema das diversas terminologias propostas para os ECC (caso-referente, história de casos, estudo retrospectivo) e cita os pontos fortes e as limitações do desenho. Em geral, são estudos mais baratos e menos demorados que os de follow up concorrentes; são especialmente úteis quando se está diante de uma doença de baixa incidência na população e com longo período de latência; permitem a avaliação de diferentes fatores que possam estar atuando separada ou conjuntamente na etiologia da doença, ou seja, têm capacidade para avaliar o efeito e as interações de um grande número de fatores que presumivelmenteestão associados com a doença sob investigação, o que torna este desenho muito atrativo; e são ainda muito úteis nas investigações cujo objetivo é a geração de hipóteses (Cole, 1979; Richardson et al., 1992).

Este texto tem como objetivo apresentar um relato sobre a utilização dos ECC até o final dos anos 80, destacando os principais aspectos na evolução do método. Em função da complexidade do tema, o texto apresenta apenas uma visão geral, que pode ser útil como ponto de partida para um maior aprofundamento. As lacunas existentes podem ser supridas pelas consultas às fontes citadas e aos manuais mais recentes, como o de Rothman \& Greenland (1998). Os aspectos relacionados ao desenho e à condução dos ECC não serão tratados.

\section{A história}

A comparação de dois grupos quanto à exposição a um fator de interesse pode ser verificada desde o século XVII, quando da real ização de estudos sobre a eficácia de distintas formas de tratamento. Lilienfeld \& Lilienfeld (1979) referem que nestes estudos já aparecia a preocupação com aspectos como randomização, necessidade de um grupo-controle, comparabilidade entre os grupos e bias (viés, distorção ou tendenciosidade associada a procedimento estatístico) de seleção, ainda que não fossem utilizados os termos e definições conhecidos nos dias atuais. Citam-se estudos sobre o tratamento do escorbuto (Lind, 1747, apud Lilienfeld \& Lilienfeld, 1979), sobre a relação entre tuberculose e hereditariedade (Louis, 1834, apud Lilienfeld \& Lilienfeld, 1979) e sobre as definições de caráter conceitual e filosófico do método comparativo (Bartlett, 1844, apud Lilienfeld \& Lilienfeld, 1979). Estes mesmos autores ressaltam a importância do trabalho de Willian Augustus Guy (1843) sobre a relação entre ocupação e saúde com particular interesse na tuberculose pulmonar. Guy definiu grupos de doentes e de não doentes, avaliou a associação com esforço físico no trabal ho, utilizando a mesma medida utilizada atualmente, a oddsratio (OR), e avaliou os vários níveis de exposição, buscando uma relação dose-reposta. Esta interpretação é, no entanto, contestada por Buck (1979), que sugere que Guy poderia estar fazendo um estudo de prevalência de tuberculose, estabelecendo comparação entre os diversos grupos ocupacionais.

A segunda metade do século XIX foi marcada pelo crescimento da bacteriologia, cuja base de sustentação implícita é hoje conhecida como teoria do germe. Este período, apesar dos inquestionáveis conhecimentos que foram pro- 
duzidos no campo das doenças infecto-contagiosas, significou o declínio da epidemiologia das "populações" e da importância atribuída às exposições ambientais, substituídas pela tentativa do controle dos agentes infecciosos. Praticamente decretou-se o fim do movimento social relacionado aos aspectos de saúde, embasado na chamada teoria dos miasmas, que floresceu na primeira metade do século, e que via nas questões econômicas e sociais a determinação do modo de adoecimento da população (Susser \& Susser, 1996a). Com a descoberta dos microorganismos, estava "estabelecido" o conhecimento a respeito da causa das doenças, o que segundo Lilienfeld \& Lilienfeld (1979) foi um importante fator para a não-realização de estudos comparativos. Não era preciso existir um grupo-controle, pois segundo os postulados de Henle-Kock, os microorganismos não estariam presentes em indivíduos sem a doença. Esta interpretação é questionada por Buck (1979), que sugere que o desenho caso-controle poderia estar na cabeça destes autores, supondo-se no referido postulado que os não casos (controles) raramente ou nunca abrigavam o microorganismo. Contudo, só com o aparecimento das primeiras vacinas foi possível retornar aos estudos comparativos.

Além de significar um freio no desenvolvimento da epidemi ologia de uma forma geral, este período pode ter significado, no particular, um certo atraso na descoberta e evolução dos ECC, na medida em que este tipo de estudo tem também aplicação no campo das doenças infecto-contagiosas, seja na avaliação de doenças agudas, seja na avaliação daquelas que seguem o caminho da cronificação. Estas últimas, praticamente em nada diferem das hoje chamadas doenças crônico-degenerativas, denominação correntemente utilizada para expressar a mai oria dos agravos de origem não infecciosa.

Nos anos 20 e 30, a epidemiologia ganhou importante contribuição vinda da sociologia, que se utilizava de estudos retrospectivos em suas investigações. Com a dificuldade para conduzir estudos experimentais, pela natureza da disciplina, os sociólogos se aprofundaram na realização de estudos observacionais com seleção de grupo-controle, pareado em função de fatores (variáveis) confundidores conhecidos. Isto ficou conhecido como Sociologia Experimental (Lilienfeld \& Lilienfeld, 1979). Este período marcou a realização dos primeiros estudos considerados como de caso-controle.

Em 1919, Broders, um cirurgião da Seção de Patologia Cirúrgica da Clínica Mayo, apresentou para a Academia de Medicina e Cirurgia de
Richmond o seu trabalho, que pode ter sido o primeiro a descrever uma associação entre hábito de fumar e desenvolvimento de carcinoma escamocelular do lábio. O autor estudou 537 indivíduos com a doença, dos quais 98\% eram do sexo masculino, e 500 homens sem câncer. Entre outros achados, verificou-se que a proporção de usuários de tabaco entre os casos foi praticamente a mesma verificada para os controles: $78,6 \%$ e $80,5 \%$, respectivamente. Um problema metodológico identificado pelo autor foi que a média de idade dos indivíduos do grupo-controle era 19 anos menor que a do grupo caso, o que poderia em parte distorcer os resultados. Porém, quando o hábito de usar cachimbo foi analisado em relação às demais formas de utilização do tabaco, encontraramse $78,5 \%$ de casos e $38,0 \%$ de controles com este hábito. Trazendo os dados do estudo para a tabela $2 \times 2$ e traduzindo-os para a atual oddsratio, podem-se verificar os seguintes resultados: uso de tabaco em geral $\mathrm{OR}=1,12$; apenas fumo $\mathrm{OR}=0,94$; hábito apenas de mascar $\mathrm{OR}=1,62$; hábito de fumar em geral e mascar $O R=2,08$; e apenas hábito de fumar cachimbo $\mathrm{OR}=7,27$. Um outro dado importante não apontado pelo autor foi a associação entre o hábito de mascar fumo e o desenvolvimento de carcinoma escamocelular do lábio, ainda que com menor magnitude do que a encontrada para o hábito de fumar cachimbo.

Em julho de 1924, George Ransey, do Departamento de Saúde de Michigan, investigou um surto de escarlatina ocorrido em Flint, Michigan. Foram obtidas histórias de 100 indivíduos, dos 116 casos notificados, que foram comparadas com as histórias de 117 indivíduos sem a doença. Dentre as diversas variáveis investigadas, o autor encontrou maior percentual de consumo freqüente de sorvete entre os casos $(60,0 \%)$ que entre os controles $(23,9 \%)$, o que teria originado uma $O R=5,34$. Seguindo a investigação, Ransey verificou que $81,9 \%$ dos consumidores utilizaram o sorvete da fábrica A, enquanto apenas $9,8 \%$ dos controles tiveram tal exposição (OR =18,9). Como visto, este autor utilizou o método caso-controle para investigar um surto de uma doença aguda, no início deste século (Ramsey, 1925).

Fonseca \& Armenian (1991) apresentaram revisão da literatura sobre a utilização de ECC para investigação de doenças agudas e demonstraram o aumento da proporção de estudos com esta metodologia, de 1:519 entre 1960/ 65 e de 1:6 entre 1980/85. Eles referiram ainda o estudo de Morales (1929, apud Fonseca \& Armenian, 1991) sobre a investigação de um surto de febre tifóide em Porto Rico, como 
um dos primeiros. Entretanto, o primeiro estudo considerado como de caso-controle foi creditado a Lane-Claypon (1926, apud Breslow \& Day, 1980), sobre o papel dos fatores reprodutivos na etiologia do câncer de mama, apesar da publicação dos estudos de Broders e de Ransey, referidos acima.

Em realidade, os ECC tomaram efetivo impulso na década de 50 , especialmente no pósguerra. Neste período, já era evidente a importância das doenças crônico-degenerativas, notadamente as doenças do coração e o câncer, que no mundo desenvolvido já causavam mais mortes que as doenças infecciosas, e que efetivamente não encontravam respostas quanto à sua etiologia com a realização de estudos de coorte.

Os epidemiologistas estavam mais uma vez, como na era dos miasmas, diante de doenças fatais cujas origens eram completamente desconhecidas. Nascia uma nova era na história da epidemiologia, a era da "epidemiologia das doenças crônicas", e com ela, os ECC. Os novos estudos tinham como objetivo estabelecer a relação entre as doenças de então e os supostos fatores causais, sem necessariamente buscar explicações quanto aos mecanismos fisiopatológicos. Este tipo de abordagem justificou a caracterização da epi demiologia como embasada no paradigma da caixa preta, cujo interior (mecanismos fisiopatológicos) é invisível aos olhos dos investigadores (Susser \& Susser, 1996a). Citam-se sobretudo os vários estudos que buscavam a relação entre o hábito de fumar e o desenvolvimento de câncer de pulmão (White, 1990). Levin et al. (1950), por intermédio do estudo de freqüências relativas, demonstraram associação entre câncer de pulmão e de lábio e utilização de fumo. O hábito de fumar cigarro aumentou marcadamente o risco de câncer de pulmão, enquanto o hábito de fumar cachimbo aumentava o risco do câncer de lábio, como já havia si do demonstrado por Broders (1920). No mesmo periódico, Winder \& Graham (1950), utilizando metodologia semel hante à de Levin et al. (1950), demonstraram a relação entre fumo e câncer de pulmão entre 684 casos de carcinoma broncogêni co e 780 controles hospitalares sem câncer. Verificaram que $96,5 \%$ dos casos e $73,7 \%$ dos controles eram fumantes, e ao buscarem a relação com a quantidade de fumo utilizada, encontraram que $51,2 \%$ dos casos e $19,1 \%$ dos controles fumavam moderada ou excessivamente. Aocorrência do carcinoma de pulmão em não fumantes foi considerada um evento raro $(2,0 \%)$. Vale ressaltar que Levin teve seu estudo inicialmente rejeitado pela revista, mas ao mesmo tempo chegou ao editor, o trabalho de Winder
\& Graham, este último renomado cirurgião que certamente não teria seu trabalho rejeitado. 0 editor resolveu então publicar os dois artigos. Para melhor apreciação deste fato ver Armenian \& Lilienfeld (1994).

Os clássicos estudos de Doll \& Hill (1950; 1952) sobre a etiologia do câncer de pulmão foram desenvolvidos com base na seleção de indivíduos entre abril de 1948 e fevereiro de 1952. Dos 3.446 pacientes com câncer neste período, os autores estudaram 1.357 homens e 108 mulheres com carcinoma de pulmão, em comparação com igual número de controles pareados por sexo, idade ( \pm 5 anos) e por hospital onde eram tratados. Concluíram pela associação entre hábito de fumar cigarros e desenvolvimento de carcinoma de pulmão entre homens $\chi^{2}=43,99$ ( $\left.p<0,000001\right)$ e entre muIheres $\chi^{2}=6,73(p<0,01)$.

Ainda que no início do século já se tenham desenvolvido medidas para quantificar o grau de associação entre variáveis numa tabela de contigência, foi Cornifield (1951) quem deu grande contribuição para o refinamento da análise dos ECC, propondo o uso da odds ratio como uma medida da estimativa do risco relativo, usando os dados dos estudos de Schrek et al . (1950) e os de Levin (1950) sobre a relação entre hábito de fumar e câncer de pulmão, eos dados de Lane-Claypon (1926, apud Breslow \& Day, 1980), sobre câncer de mama. Este autor propôs um método para se estimar a taxa de incidência da doença entre expostos e entre não expostos ao fator sob investigação, já que os estudos que utilizou eram baseados no método comparativo, valendo-se dos casos e verificando a presença ou ausência do fator em termos percentuais, de forma retrospectiva. Segundo Cornifield, estetipo de desenho não permitia o cálculo de uma taxa de incidência verdadeira, mas apenas uma freqüência relativa.

Assumindo que a doença estudada seja rara na população geral, é possível aceitar como verdade que as diferenças encontradas nas freqüências relativas do fator entre doentes e não doentes será diferente de um. Porém, usandose a taxa de incidência da doença na população geral na fórmula proposta, calculam-se as taxas de incidência da doença entre expostos e não expostos. Utilizando os dados de Schrek, Cornifield (1951) construiu então a razão entre as duas taxas e concluiu que a incidência da doença entre os expostos era 2,5 vezes maior que entre os não expostos.

Reconhecendo que a maioria dos estudos clínicos utiliza um número limitado de casos, Cornifield referiu ainda a importância de se estimar os limites de erro da razão calculada e 
propôs o cálculo dos intervalos de confiança. Se a taxa de incidência da doença na população estiver fora do intervalo, conclui-se que há uma diferença significativa entre expostos e não expostos. Alerta, todavia, que para se chegar a esta conclusão, tanto o grupo de doentes quanto o grupo-controle devem ser representativos da população que os gerou. Chamou a aten ção principalmente para as diferenças na distribuição etária. O pressuposto da raridade da doença foi bastante discutido posteriormente, e ficou claro que esta necessidade depende do tipo de desenho de estudo que se vai conduzir (Greenland \& Thomas, 1982; Greenland et al., 1986; Miettinen, 1976; Pearce, 1993). Rodrigues \& Kirkwood (1990) oferecem ótima revisão sobre a utilização de estudos caso-controle para doenças não raras.

Mantel \& Haenszel (1959) estudaram os aspectos estatísticos da anál ise de dados de estudos retrospectivos com o objetivo primário de extrair deles as mesmas conclusões que seriam obtidas em um estudo prospectivo, caso este houvesse sido realizado. Avançaram no sentido oposto, para mostrar que em algumas situações pode ser útil uma avaliação retrospectiva de dados já coletados prospectivamente. Possivelmente esta foi uma das primeiras proposições do que hoje se conhece como estudo caso-controle aninhado, cujo desenvolvimento ocorre no interior de uma coorte sob acompanhamento, utilizando-se como casos os indivíduos que se tornaram doentes no período, e como controles os demais (todos os indivíduos, uma amostra, ou indivíduos pareados). Discutiram datalhadamente os cuidados que devem ser tomados quando da seleção dos indivíduos do grupo-controle, particularmente se este é originário da população hospitalizada, enfatizando o caráter representativo dos casos e dos controles em relação à população geral, a utilização de restrição e pareamento para controle de variáveis confundidoras, bem como as dificuldades apresentadas quando da utilização desta técnica. Uma grande contribuição destes autores foi o desenvolvimento do cál culo da odds ratio sumarizada $\left(\mathrm{OR}_{\mathrm{MH}}\right) \mathrm{e}$ do qui-quadrado sumarizado utilizado quando o(s) fator(es) sob investigação se apresenta(m) com dois ou mais níveis.

Outro avanço no campo analítico surgiu neste período com a proposição de Levin (1953, apud Coughlin et al., 1994) para o cálculo do risco atribuível. Na década de 70, MacMahon \& Pugh (1970) e Cole \& M acMahon (1971) apresentaram método para se calcular o risco atribuível percentual com base num ECC. Miettinen (1976) e Neutra \& Drolette (1978) propuse- ram formas de se calcular a fração etiológica nos ECC, o primeiro para os ECC de densidade de incidência, e o segundo para osECC de incidência cumulativa e ECC tipo prevalência, utilizando o teorema de Bayes. Uma revisão sobre risco atribuível, dirigida aos ECC, pode ser encontrada em Coughlin et al. (1994).

Nenhum assunto a respeito dos ECC mobilizou mais os estudiosos do que a ocorrência de bias. Cornifield \& Haenszel (1960) chamavam a aten ção para os possíveis erros presentes em um estudo retrospectivo, mas, 14 anos antes, Berkson (1946) já publicara importante trabalho cujo objetivo foi mostrar as limitações da aplicação da tabela $2 \times 2$ quando da utilização de dados originados de população hospitalizada. Demonstrou que cada doença na população geral tem uma probabilidade definida de resultar em internação, e de que estas probabilidades seletivas atuam de forma independente para cada doença. Berkson chegou à conclusão de que a probabilidade de um indivíduo com mais de uma doença ser internado é crescente (duas doenças, duas vezes; três doenças, três vezes e assim sucessivamente), o que caracteriza a população hospitalar como não representativa da população geral. Este tipo de problema, posteriormente conhecido como bias, paradoxo ou falácia de Berkson, foi alvo de atenção e de intenso debate.

Feinstein (1973), um dos mais incisivos críticos do desenho caso-controle, publicou artigo no qual listou todas as possíveis fraquezas do método, quase que inviabilizando-o do ponto de vista científico. Referiu os dizeres de Cornifield (1951) e de Mantel \& Haenszel (1959) quanto aos cuidados que um investigador deve ter ao realizar um estudo retrospectivo, como citações que mostravam a grande debilidade do método, quando na realidade, pelo que se pode abstrair dos textos, aqueles autores tinham como objetivo buscar formas de aprimorar este tipo de desenho. Foi mais adiante e criticou duramente a forma de se calcular a estimativa do risco relativo proposta por Cornifield (1951), ao assumir o pressuposto da raridade da doença. No entanto, este artigo é mais conhecido pelo fato de o autor ter criado o termo "trohoc", em contraposição ao termo "cohort", após ter feito exaustiva pesquisa para sugerir um nome que, a seu ver, melhor designasse um estudo que parte da definição de doentes e de não doentes e busca a história pregressa de exposição. Este termo foi posteriormente caracterizado como pejorativo (Sartwell, 1979) e que em nada contribuía para o avanço do método. Feinstein, entretanto, parece ter se rendido aos atrativos oferecidos pelo método e par- 
ticipou da realização de um ECC que avaliou a proteção do uso de estrógenos contra fraturas do rádio e do quadril na menopausa (Hutchinson et al ., 1979).

Um dos artigos que podem ser incluídos entre os clássicos, referentes aos ECC foi publicado por Miettinen (1985), no qual o autor discutiu a essência deste tipo de estudo e propôs princípios básicos para a seleção dos indivíduos. Posicionou-se contrariamente ao conceito freqüentemente divulgado de que o ECC é o oposto do estudo de coorte e cunhou a expressão falácia do trohoc, em contraposição ao proposto por Feinstein (1973). Da mesma forma concluiu que o ECC não é uma simples alternativa ao estudo de coorte, mas ao contrário, comporta uma estratégia própria de investigação que parte do censo e amostragem (case base) de uma população para a verificação dos fatos por esta experimentados (study base).

Horwitz (1987) propôs que os ECC seguissem o paradigma dos estudos clínicos randomizados, já que os "estudos caso-controle seguem a direção inversa da lógica científica". Mayes et al. (1988) foram adiante, levantando 56 tópicos com resultados contraditórios em uma extensa lista de ECC. Esta publicação gerou uma resposta veemente de Cumming \& Kelsey (1988), na qual questionaram o rigor científico utilizado por aqueles autores; levantaram 10 falhas daquel a publicação e concluíram que, quando realizado adequadamente, os ECC são uma ferramenta poderosa para a investigação epidemiológica. Aquela visão foi também rebatida por M iettinen (1989), quando afirmou que a perspectiva do ECC é incompatível com o paradigma dos estudos clínicos randomizados, enfatizando ainda a importância da avaliação não apenas da exposição, mas também da não-exposição. Sacket (1979) apresentou um catálogo de bias que podem ser encontrados nos ECC, mas Wünsch Filho (1992) assinala, no entanto, que dificilmente estes bias aparecem em sua forma pura. Kopec \& Esdaile (1990), ao procederem à extensa revisão sobre bias, concluíram que em ótimas condições, os ECC são tão válidos quanto os estudos clínicos randomizados.

Cole (1979) lembra uma importante questão quanto ao aspecto temporal dos ECC. Não há que se rebaixar o valor deste desenho porque se trata de uma ol hada para trás, da doença para a exposi ção, o caminho inverso, antinatural. Ao contrário, deve-se lembrar que eventos do dia a dia são vistos desta forma, e nem por isto são difíceis de serem entendidos. Ressalte-se que estas pequenas relações do cotidiano são geral mente de curta duração. Por- tanto, quando se está estudando a relação entre uma exposição e uma doença, com um período de tempo longo entre eles, as vezes com uma fraca associação, uma ferramenta especial é o estudo de caso-controle.

Com o crescimento e evolução dos ECC, muitas vezes envoltos em discussões sobre as limitações inerentes ao próprio método, foram realizados importantes fóruns de debate sobre o tema. Em dezembro de 1977, 20 epidemiologistas e estatísticos participaram de um work shop promovido pela International Agency for Research on Cancer (IARC) com o objetivo de discutir os aspectos estatísticos dos ECC, o que culminou com a publicação, ainda hoje bastante atual, do Statistical Methods in Cancer Research, que se inclui entre os principais livros sobre o assunto (Breslow \& Day, 1980). Em abril de 1978 foi realizado nas Bermudas o Simpósio sobre os Estudos Caso-Controle, ondeforam discutidos a história, a evolução, os métodos e estudos ilustrativos. Este evento, marcado pela confrontação de idéias divergentes sobre os ECC, concluiu que este desenho é de grande importância para a investigação epidemiológica e recomendou, reconhecendo a sua susceptibilidade aos bias, um aprimoramento da técnica. Em 1979 foi publicada uma edição especial do Journal of Chronic Diseases, inteiramente dedicada às discussões travadas neste encontro. Baseado nesta publicação, I brahim (1979) Iançou o livro Case-Control Studies: Consensus and Controversy, e Schelleselman (1982) publicou um livro exclusivamente dedicado aos ECC, que se constitui ainda hoje em importante fonte sobre o tema.

A crescente utilização dos ECC na década de 80 justificou a edição especial do Epidemiologi c Reviews com uma ampla revisão sobre o método. Nesta edição, além da necessidade sempre presente de se aprimorar o método, apontam-se as novas perspectivas dos ECC com a utilização dos marcadores biológicos, que permitem uma avaliação mais precisa da exposição e da doença. Ressalta-se que, no futuro, os ECC devem tornar-se mais sensíveis à detecção de associações fracas e moderadas e que os estudos aninhados a uma coorte oferecerão uma boa alternativa para conjugar as vantagens de ambos os desen hos, notadamente quando se estiver diante de caros ensaios biológicos (Austin et al., 1994).

\section{Conclusões}

É interessante notar que os ECC compõem hoje um cenário no qual protagonizam um curio- 
so paradoxo, em decorrência do seu uso cada vez maior em investigações com os marcadores biológicos. Desconhecidos na era da epidemiologia das doenças infecciosas, nascidos praticamente na era da epidemiologia das doenças crônico-degenerativas, sob o paradigma da caixa preta, com sua similitude ao paradigma dos miasmas, os ECC são cada vez mais importantes nas investigações epidemiológicas em nível molecular, contribuindo para a "abertura da caixa" e talvez para o ressurgimento de uma "nova teoria do germe" (Loomis \&Wing, 1990). Susser \& Susser (1996b) referem o momento atual como de transição para a era da eco-epidemiologia, com o seu paradigma das caixas chinesas, que representam um con-

\section{Agradecimentos}

À Coordenação de Aperfeiçoamento de Pessoal de Nível Superior; à School of Public Health/University of North Carolina at Chapel Hill, Estados Unidos; à Fogarty International Center, e aos Drs. Naomar de Almeida Filho e Rita de Cássia Franco Rêgo - Instituto de Saúde Coletiva/Universidade Federal da Bahia, pelos comentários.

\section{Referências}

ACHESON, E. D., 1979. Comment. Journal of Chronic Diseases, 32:28-29.

ARMENIAN, H. K. \& LILIENFELD, D. E., 1994. Overview and historical perspective. Epidemiologic Reviews, 16:1-5.

ARMENIAN, H. K. \& GORDIS, L., 1994. Future perspectives on the case-control method. Epidemiologic Reviews, 16:163-164.

AUSTIN, H.; HILL, H. A.; FLANDERS, W. D. \& GREENBERG, R. S., 1994. Limitations in the application of case-control methodology. Epidemiologic Reviews, 16:65-76.

BERKSON, J., 1946. Limitations and application of fourfold table analysis to hospital data. Biometrics Bulletin, 2:47-53.

BRESLOW, N. E. \& DAY, N. E., 1980. Statistical Methods in Cancer Research: The Analysis of Case-control Studies. v. 1, Scientific Publication 32. Lyon: International Agency for Research on Cancer.

BRODERS, A. C., 1920. Squamous-cell epithelioma of the lip. JAMA, 74:656-664.

BUCK, C., 1979. Comment. Journal of Chronic Diseases, 32:14.

CLARKE, E. A. \& ANDERSON, T. W., 1979. Does screening by "pap" smears help prevent cervical cancer? A case-control study. Lancet, 2:1-4.

COLE, P. \& MacMAHON, B., 1971. Attributable risk percent in case-control studies. British Journal of Preventiveand Social Medicine, 25:242-244. junto de níveis hierarquizados que inter-relaciona os ambientes social e biológico, desde o nível mais macro (físico, ambiental) até o nível molecular. Logo, seja qual for o rumo da epidemiologia, claro está o papel dos estudos casocontrole, talvez como o carro-chefe das investigações epidemiológicas. No dizer de Armenian \& Gordis (1994), os ECC chegaram à idade adulta. Anos atrás, o método caso- controle era visto como um mau necessário e considerado o segundo melhor. Hoje, no entanto, os ECC oferecem contribuição singular às investigações etiológicas e às de avaliação, e se constituem em importante instrumento para o desenvolvimento das ações de saúde pública.
COLE, P., 1979. The evolving case-control study. Journal of Chronic Diseases, 32:15-27.

COMSTOCK, G. W., 1994. Evaluating vaccination effectiveness and vaccine efficacy by means of casecontrol studies. Epidemiologic Reviews, 16:77-89.

CORNFIELD, J. A., 1951. Method of estimating comparative rates from clinical data: Applications to cancer of the lung, breast and cervix. Journal of theN ational Cancer Institute, 11:1269-1275.

CORNFIELD, J. \& HAENSZEL, W., 1960. Some aspects of retrospective studies. Journal of Chronic Diseases, 11:523-534.

COU GHLIN, S. S.; BENICHOU, J. \&WEED, D. L., 1994. Attributable risk estimation in case-control studies. Epidemiologic Reviews, 16:51-64.

CUMMING, R. L. \& KELSEY, J. L., 1988. Case-control studies. International Journal of Epidemiology, 17:725-726.

DOLL, R. \& HILL, A. B., 1950. Smoking and carcinoma of the lung: Preliminary report. BMJ, 2:739-748.

DOLL, R. \& HILL, A. B., 1952. A study of the aetiology of carcinoma of the lung. BMJ, 2:1271-1286.

FEINSTEIN, A. R., 1973. The epidemi ologic TROHOC, the ablative risk ratio, and 'retrospective' research. Clinical Pharmacology and Therapeutics, 14:291-307.

FONSECA, M. G. P. \&ARMENIAN, H. K., 1991. Use of the case-control method in outbreak investigations. American Journal of Epidemiology, 133:748-752. 
GREENLAND, S. \&THOMAS, D. C., 1982. On the need for the rare disease assumption in case-control studies. American Journal of Epidemiology, 116: 547-553.

GREENLAND, S.; THOMAS, D. C. \& MORGENSTERN, $\mathrm{H} .$, 1986. The rare disease assumption revisited. A critique of "estimators of relative risk for casecontrol studies". American Journal of Epidemiology, 124:869-876.

GUY, W. A., 1843. Contributions to a knowledge of the influence of employment on health. Journal of the Royal Statistics Society, 6:197-211.

HORWITZ, R. I., 1987. The experimental paradigm and observational studies of cause-effect relationships in clinical medicine. Journal of Chronic Diseases, 40:91-99.

HUTCHINSON, T. A.; POLANSKY, S. M. \& FEINSTEIN, A. R., 1979. Post-menopausal estrogens protect against fractures of hip and distal radius. A casecontrol study. Lancet, 2:705-709.

IBRAHIM, M. A., 1979. The Case-Control Study:Consensus and Controversy. Oxford: Pergamon Press.

KOPEC, J. A. \& ESDAILE, J. M., 1990. Bias in case-control studies. A review. Journal of Epidemiology and Community Health, 44:179-186.

LEVIN, M. L.; GOLDSTEIN, H. \& GERHARDT, P. R., 1950. Cancer and tobacco smoking: Preliminary report. JAMA, 143:336-338.

LILIENFELD, D. E., 1979. "The greeting of Epidemiology": Sanitary physicians and the London Epidemiological Society (1830-1870). Bulletin of the History of Medicine, 52:503-528.

LILIENFELD, A. M. \& LILIENFELD, D. E., 1979. A century of case-control studies: Progress? Journal of Chronic Diseases, 32:5-13.

LOOMIS, D. \&WING, S., 1990. Is molecular epidemiology a germ theory for the end of the twentieth century? International Journal of Epidemiology, 19:1-3.

MacMAHON, B. \& PUGH, T. F., 1970. Epidemiology, Principles and Methods. Boston: Little Brown.

MANTEL, N. \& HAENSZEL, W., 1959. Statistical aspects of the analysis of data from retrospective studies of disease. Journal of the National Cancer Institute, 22:719-748.

MAYES, L. C.; HORWITZ, R. I. \& FEINSTEIN, A. R., 1988. Collection of 56 topics with contradictory results in case-control research. International Journal of Epidemiology, 17:680-685.

MIETTINEN, O. S., 1976. Estimability and estimation in case-referent studies. American Journal of Epidemiology, 103:225-235.

MIETTINEN, O. S., 1985. The "case-control" study: Valid selection of subjects. Journal of Chronic Diseases, 38:543-548.

MIETTINEN, O. S., 1989. Unlearned lessons from clinical trials: A duality of outlooks. Journal of Clinical Epidemiology, 42:499-502.

NEUTRA, R. R. \& DROLETTE, M. E., 1978. Estimating exposure-specific disease rates from case-control studies using Bayes' theorem. American Journal of Epidemiology, 106:214-222.

NORONHA, C. P.; STRUCHINER, C. J. \& HALLORAN, M. E., 1995. Assessment of the direct effectiveness of $B C$ meningococcal vaccine in Rio de Janeiro, Brazil: A case-control study. International Journal of Epidemiology, 24:1050-1057.
PEARCE, N., 1993. What does the odds ratio estimate in a case-control study? International Journal of Epidemiology, 22:1189-1192.

RAM SEY, G. H., 1925. An epidemic of scarlet fever spread by ice cream. American Journal of Hygiene, 25:669-681.

RICHARDSON, S.; ZITTOUN, R.; BASTUJI-GARIN, S.; LASSERRE, V.; GUIHENNEUC, C.; CADIOU, M.; VIGUIE F. \& LAFFONT-FAUST, I., 1992. Occupational risk factors for acute leukaemia: A casecontrol study. International Journal of Epidemiology, 21:1063-1073.

RODRIGUES, L. \& KIRKWOOD, B. R., 1990. Case-control designs in the study of common diseases: Updates on the demise of the rare disease assumption and the choice of sampling scheme for controls. International Journal of Epidemiology, 19: 205-213.

ROTHMAN, K. J. \& GREENLAND, S., 1998. Modern Epidemiology. 2nd Ed. Philadelphia: Lippincott Raven.

SACKET, D. L., 1979. Bias in analytic research. Journal of Chronic Diseases, 32:51-63.

SARTWELL, P. E., 1979. Comment. Journal of Chronic Diseases, 32:42-44.

SCHLESSELM AN, J. J., 1982. Case-Control Studies Design,Conduct, Analysis. New York/Oxford: Oxford University Press.

SCHREK, R.; BAKER, L. A.; BALLARD, G. P. \&DOLGOFF, S., 1950. Tobacco smoking as an etiologic factor in disease. I. Cancer. Cancer Research, 10:49-58.

SELBY, J. V., 1994. Case-control evaluations of treatment and program efficacy. Epidemiologic Reviews, 16:90-101.

SUSSER, M. \& SUSSER, E., 1996a. Choosing a future for Epidemiology: I. Eras and paradigms. American Journal of Public Health, 86:668-673.

SUSSER, M. \& SUSSER, E., 1996b. Choosing a future for Epidemiology: II. From black box to Chinese boxes and Eco-Epidemiology. American Journal of Public Health, 86:674-677.

TSAI, Y. J.; WANG, J. D. \& HUANG, W. F., 1995. Casecontrol study of the effectiveness of different types of helmets for the prevention of head injuries among motorcycle riders in Taipei, Taiwan. American Journal of Epidemiology, 142:974-981.

WEISS, N. S., 1994. Application of the case-control method in the evaluation of screening. Epidemiologic Reviews, 16:102-108.

WHITE, C., 1990. Research on smoking and lung cancer: A landmark in the history of chronic disease epidemiology. Yale Journal of Biology \& Medicine, 63:29-46.

WÜNSCH FILHO, V., 1992. Riscos Ocupacionais eCâncer dePulmão. Tese de Doutorado, São Paulo: Departamento de Epidemiologia, Faculdade de Saúde Pública, Universidade de São Paulo.

WYNDER, E. L. \& GRAHAN, E. A., 1950. Tobacco smoking as a possible etiological factor in bronchiogenic carcinoma. JAMA, 143:329-336. 